\title{
Predictive factors for corrective effect of inferior rectus recession for congenital superior oblique palsy
}

\section{AUTHOR(S):}

Miyata, Manabu; Shibata, Kiyo; Hamasaki, Ichiro; Hata, Masayuki; Muraoka, Yuki; Yoshikawa, Munemitsu; Hasebe, Satoshi; Ohtsuki, Hiroshi

\section{CITATION:}

Miyata, Manabu ...[et al]. Predictive factors for corrective effect of inferior rectus recession for congenital superior oblique palsy. Graefe's Archive for Clinical and Experimental Ophthalmology 2018, 256(2): 403-409

\section{ISSUE DATE:}

2018-02

URL:

http://hdl.handle.net/2433/230337

\section{RIGHT:}

This is a post-peer-review, pre-copyedit version of an article published in 'Graefe's Archive for Clinical and Experimental Ophthalmology'. The final authenticated version is available online at: http://dx.doi.org/10.1007/s00417-017-3838-z,

The full-text file will be made open to the public on 01 February 2019 in accordance with publisher's 'Terms and Conditions for Self-Archiving'.; この論文は出版社版でありません。引用の際には出版社版をご確認ご利用ください。 This is not the published version. Please cite only the published version. 
Predictive Factors for Corrective Effect of Inferior Rectus Recession for Congenital Superior Oblique Palsy

MANABU MIYATA ${ }^{1 *}$, KIYO SHIBATA ${ }^{2}$, ICHIRO HAMASAKI $^{2}$, MASAYUKI HATA $^{1}$, YUKI MURAOKA ${ }^{1}$, MUNEMITSU YOSHIKAWA ${ }^{1}$, SATOSHI HASEBE ${ }^{3}$, and HIROSHI OHTSUKI ${ }^{2}$

${ }^{1}$ Department of Ophthalmology and Visual Sciences, Kyoto University Graduate School of Medicine, Shogoin Kawahara-cho 54, Sakyo-ku, Kyoto, Japan

2Department of Ophthalmology, Okayama University Graduate School of Medicine, Dentistry, and Pharmaceutical Sciences, Shikata-cho 2-5-1, Kita-ku, Okayama, 700-8558, Japan

32Department of Ophthalmology 2, Kawasaki-Hospital, Kawasaki Medical School, Nakasange 2-1-80, Kita-ku, Okayama, 700-8505, Japan

\section{Corresponding Author:}

Manabu Miyata

Department of Ophthalmology and Visual Sciences, Kyoto University Graduate School of Medicine

Shogoin Kawahara-cho 54, Sakyo-ku, Kyoto 606-8507, Japan

Phone: 011-81-75-751-3248

Fax: 011-81-75-752-0933

Email: miyatam@kuhp.kyoto-u.ac.jp 


\section{ABSTRACT}

\section{- Purpose:}

To identify preoperative factors associated with the surgical corrective effect of contralateral inferior rectus recession (IRR) for vertical deviation in patients with congenital superior oblique palsy (SOP).

\section{- Methods:}

This retrospective study included 20 treatment-naïve patients with unilateral congenital SOP (age range, 6-79 years) who underwent contralateral IRR according to our basic policy to select IRR for paretic eye fixation. The corrective effect $(\% / \mathrm{mm})$ of IRR was defined as the difference in the vertical deviation at the primary gaze position between before and 6-18 months after surgery per distance of recession. We also measured the preoperative vertical deviation at primary and secondary gaze positions, and vertical deviation with head-tilting, and calculated the difference in vertical deviation between these positions. We analyzed the correlation between the corrective effect of IRR and these study parameters.

\section{- Results:}

The mean corrective effect of IRR was $2.4 \pm 1.6^{\circ} / \mathrm{mm}$, which had a significant correlation with preoperative differences in vertical deviation between the primary gaze position and the downward $(P=0.004, r=-0.61)$ and contralateral gaze positions $(P=0.03, r=-0.48)$; and the presence of preoperative stereopsis $(P=0.02, r=-0.51)$. After excluding a statistical outlier, the correlation between the corrective effect and the difference between the primary and contralateral gaze positions was no longer significant $(P=0.07)$, while the other two relationships remained significant.

\section{- Conclusions:}

Our findings suggest that preoperative differences in vertical deviation between the primary and downward gaze positions and the presence of preoperative stereopsis are important considerations prior to performing IRR for congenital SOP, particularly with paretic eye fixation.

\section{KEYWORDS}

superior oblique palsy; inferior rectus recession; corrective effect; vertical deviation; predictive factor 


\section{INTRODUCTION}

Contralateral inferior rectus recession (IRR) is an option for the surgical treatment for patients with congenital superior oblique palsy (SOP). Knapp classified SOP into five patterns according to a grading system based on the positions of greatest vertical misalignment [1, 2]. Knapp's classifications system is widely used for the selection of surgical procedure for SOP [3-6]; therefore, IRR is performed less frequently than other procedures. For example, in a report by von Noorden et al., IRR was selected to treat SOP in only $9 \%$ of cases, whereas ipsilateral inferior oblique recession (IOR) was used in 56\% [3]. However, Miyata et al. reported that IRR, when selected on the basis of eye fixation, improved excyclodeviation in the vertical and horizontal gaze positions of patients with congenital SOP [7]. Furthermore, this procedure is easier to perform than IOR because the insertion of the inferior oblique (IO) muscle is deep and the vortex vein is often close to the 10 muscle. However, a significant concern of using IRR for treating SOP is the instability of the surgical corrective effect on vertical deviation. Therefore, the preoperative prognostic factors associated with the corrective effect of IRR need to be elucidated.

In most patients with monocular congenital SOP, the strabismic eye is the paretic eye. Thus, IOR of the paretic eye is a reasonable treatment choice. However, some patients, particularly adults with decompensated palsy, exhibit paretic eye fixation. Jiang and Demer reported that contralateral IR contracture is observed in patients with unilateral SOP using high-resolution magnetic resonance images (MRI) [8]. This finding may result from the effects of long-term palsy, including patients with paretic eye fixation. Dickey et al. reviewed that contracture of the contralateral inferior rectus (IR) muscle develops in cases of unilateral SOP where fixation occurs in the paretic eye because of prolonged hypodeviation of the non-paretic eye [9]. This IR contracture would influence the corrective effect of IRR. However, to what degree IR contracture impacts the corrective effect of IRR with respect to eye fixation has not been determined. The corrective effect of IRR is, therefore, difficult to predict before surgery.

Von Noorden et al. reported the corrective effect of SOP surgery for the nine cardinal positions of the gaze according to Knapp's recommendations [3]. They demonstrated that the corrective effect of IRR on vertical deviation was 4.82, 8.91, and 17.45 prism diopters in the upward, primary, and downward gaze positions, respectively. However, preoperative vertical deviation differed between the upward and downward gaze positions because the majority of cases studied were Knapp class V. Because IRR is often performed for Knapp class $V$ cases [10], the corrective effect of IRR for other SOP cases, such as those with similar vertical deviation in the upward and downward gaze positions, has not been determined. 
In this study, we investigated the corrective effect of IRR, with reference to eye fixation. Furthermore, we investigated the preoperative factors associated with the surgical corrective effect of IRR on vertical deviation, by considering vertical deviation in the primary and secondary gaze positions.

\section{MATERIALS AND METHODS}

This retrospective cohort study was approved by the Institutional Review Board (IRB) at Okayama University Graduate School of Medicine, Dentistry, and Pharmaceutical Sciences (Okayama, Japan). All study protocols adhered to the tenets of the Declaration of Helsinki. For treatment, written informed consent was obtained from each patient or guardian. According to our IRB guidelines, informed consent was not needed for retrospective review of the patients' medical records.

\section{Subjects}

Consecutive treatment-naïve Japanese patients $(n=131)$ who underwent vertical muscle surgery for unilateral congenital SOP, who visited the Department of Ophthalmology at Okayama University Graduate School of Medicine, Dentistry, and Pharmaceutical Sciences (Okayama, Japan) between June 2008 and May 2013, were initially enrolled in this study. All patients underwent comprehensive ophthalmological and strabismological examinations, including measurement of best-corrected visual acuity (BCVA) using a decimal visual acuity chart (Landolt chart); indirect ophthalmoscopy; slit-lamp biomicroscopy; measurement of stereoacuity using the TNO stereo test (Lamèris Ootech BV Nieuwegein, the Netherlands); prism and alternating cover testing at $5 \mathrm{~m}$ and $0.3 \mathrm{~m}$; right and left head tilt test; binocularity test using Bagolini striated glasses; and ocular motility assessment. Unilateral congenital SOP, including decompensated SOP, was diagnosed using strabismological examinations (Kushner's recommendation) [11], medical interview, and vertical fusion range by strabismus specialists. The inclusion criteria included patients with unilateral congenital SOP who underwent contralateral IRR. The exclusion criteria included the presence of dissociated vertical deviation, a previous history of head injury, masked bilateral SOP, or absence of assessable data. In total, 20 patients (10 women and 10 men) with SOP were included in the final analyses.

\section{Surgical Procedures}

According to our standard policy for treating treatment-naïve patients with SOP, the strabismic eye was selected for vertical muscle recession, i.e. ipsilateral IOR for cases with a fixation preference for the non-paretic eye, and contralateral IRR for 
cases with a fixation preference for the paretic eye. However, we occasionally perform IOR for cases with a fixation preference for the paretic eye, to correct overaction of the inferior oblique muscle (IO-OA) in the paretic eye, and IRR for cases with a fixation preference for the non-paretic eye, because of the absence of IO-OA and/or small vertical deviation. However, we did not use superior oblique tuck in any case, because it is well known to induce Brown syndrome [12]. The IR muscle was fixated using three knots of 7-0 nylon to prevent muscle slippage without horizontal transposition, and we did not use adjustable sutures to prevent late overcorrection [13]. We determined the distance of recession by $3(\Delta / \mathrm{mm})$ based on the targeted vertical deviation measured using a prism adaptation test (PAT), because Ohtsuki et al. reported the usefulness of PAT in surgery for SOP [14]. All surgeries in patients aged $\leq 12$ years were performed under general anesthesia, whereas local anesthesia was used for all patients aged $\geq 13$ years. The criterion for surgical motor success was a vertical deviation of $\leq 5 \Delta$, according to a previous report [15].

\section{Analysis of the Corrective Effect of IRR and Preoperative Vertical Deviation}

We measured vertical deviation using prism and alternating cover testing. We analyzed the corrective amount $\left(^{\circ}\right)$ of IRR as the difference in vertical deviation at the primary gaze position at $5 \mathrm{~m}$ between before and 6-18 months after surgery. The 'corrective effect' $\left({ }^{\circ} / \mathrm{mm}\right)$ of IRR was defined as the corrective amount $\left(^{\circ}\right)$ per unit distance of recession ( $\mathrm{mm}$ ). Preoperative vertical deviation was determined for the five gaze positions (primary and secondary gaze positions): primary, upward, downward, contralateral, and ipsilateral gaze positions. We calculated the difference in vertical deviation between the primary and secondary gaze positions; positive values equated to a greater vertical deviation in the secondary gaze position than the primary gaze position. We also determined vertical deviation during head-tilting and calculated the difference between vertical deviation when tilting to the contralateral and ipsilateral sides.

\section{Statistical Analysis}

Data are presented as mean \pm standard deviation, where applicable. All BCVA data were converted to the logarithm of the minimal angle of resolution (logMAR) for statistical analyses. All prism diopters were converted to degrees for statistical analyses. Correlation analysis was performed using Spearman's rank correlation coefficients to analyze relationships between the corrective effect of IRR and preoperative parameters including the following: age, sex, logMAR VA of eyes operated on, presence of stereopsis (stereoacuity $\leq 1980$ sec of arc: 0 ; unmeasurable stereoacuity by TNO: 1), distance of recession, duration between surgery and 
measurement, vertical deviation in the primary gaze position at $5 \mathrm{~m}$ with the head tilted to the contralateral and ipsilateral sides, difference between vertical deviation when the head was tilted to the contralateral and ipsilateral sides, vertical deviation in the primary, upward, downward, contralateral, and ipsilateral gaze positions at $5 \mathrm{~m}$, and difference in vertical deviation between the primary and secondary gaze positions. Statistical outliers were defined as values smaller than the lower quartile $1.5 \times$ interquartile range (IQR), or larger than the upper quartile $+1.5 \times$ IQR. For each parameter, correlation analysis was performed once using the entire data set, and then again with the outliers excluded. All statistical analyses were performed using SPSS statistics software version 21 (IBM Corp., Armonk, NY). A P-value of $<0.05$ was considered statistically significant.

\section{RESULTS}

The characteristics of the study population are summarized in Table 1 . The patient age was $58.8 \pm 18.7$ years (range, 6 to 79 years). Postoperative data was obtained $355 \pm 88$ days after surgery. The prevalence of paretic eye fixation was $85 \%(17 / 20)$. The preoperative vertical deviation in the primary gaze position at $5 \mathrm{~m}$ was $10.9 \pm 5.4^{\circ}$. The distance of recession was $3.7 \pm 1.0 \mathrm{~mm}$. The corrective effect of IRR in the primary gaze position was $2.4 \pm 1.6^{\circ} / \mathrm{mm}$ (Figure 1 ). One outlier of $7.3 \% / \mathrm{mm}$ was detected. The surgical motor success rates in the primary, upward, downward, contralateral, and ipsilateral gaze positions were 70\% (14/20), 80\% (16/20), 55\% $(11 / 20), 65 \%(13 / 20)$, and 65\% (13/20), respectively. Reoperation was performed in $15 \%(3 / 20)$ during the follow-up period.

\section{Correlations between the Corrective Effect of IRR on Vertical Deviation and Preoperative Study Parameters}

The correlations between the corrective effect of IRR and preoperative study parameters are presented in Table 2. We found significant negative correlations between the corrective effect of IRR; and preoperative differences in vertical deviation between the primary gaze position and the downward $(P=0.004, r=-0.61$, Figure 2$)$ and contralateral gaze positions $(P=0.03, r=-0.48)$, as well as the presence of preoperative stereopsis $(P=0.02, r=-0.51)$. There were no other significant correlations. When we excluded the outlier, the analysis confirmed a significant correlation between corrective effect; and preoperative differences in vertical deviation between the primary gaze position and the downward gaze position, and the presence of preoperative stereopsis $(P=0.01,-0.56 ; P=0.049, r=-0.46$, respectively). However, there was no longer a significant correlation between the 
corrective effect and preoperative difference between the primary and contralateral gaze positions $(P=0.07)$.

\section{DISCUSSION}

The present study demonstrated that the corrective effect of IRR on vertical deviation for congenital SOP, for which the surgical procedure was selected on the basis of eye fixation, was strongly correlated with the preoperative difference in vertical deviation between the primary and downward gaze positions $(P=0.004, r=-0.61)$. Hence, smaller differences in preoperative vertical deviation in the downward gaze position, compared to the primary gaze position, are associated with a greater corrective effect for IRR. This result is rational, considering that the action of the contractured IR muscle is weak in the direction of the muscle action and that contracture has previously been shown to induce a high corrective effect in thyroid-associated ophthalmopathy [16]. Because our results suggest that the surgical outcomes of IRR can be predicted preoperatively, we recommend performing prism and alternating cover testing prior to surgery to compare differences in vertical deviation between the primary and downward gaze positions. Thus, we propose, as per Figure 2, the selection of surgical procedures other than IRR in cases with markedly less vertical deviation in the downward gaze position than in the primary gaze position.

We found that the corrective effect was larger in cases with preoperative stereopsis of $\leq 1980 \mathrm{sec}$ of arc. The postoperative vertical deviation was possibly underestimated in cases with stereopsis due to strong fusion. Simonsz et al. found using the Bielschowsky head-tilt test, that the ratio of change in vertical deviation against the amplitude of ocular counterrolling was higher in long-standing palsies, compared to short-standing palsies [17]. In the present study, patients with high binocularity could have had potentially decreased vertical deviation using slight head tilting, an adaptation that would not have been detected by investigators. A 30-45 min patch test could help measure the patients' deviation more accurately than the prism and alternate cover testing we performed.

In the present study, we found that the average corrective effect of IRR on vertical deviation in the primary gaze position was $2.4 \pm 1.6 \% \mathrm{~mm}$, which is greater than that reported for IRR for the correction of hypotropia following cataract extraction $(1.7 \pm 1.3 \% \mathrm{~mm})$ [18]. This is possibly owing to greater contracture of the IR muscle in the numerous patients with SOP with paretic eye fixation in the present study (85\%). Using MRI, Jiang and Demer observed that, in unilateral SOP, the contralateral IR muscle was larger and more contractile than the ipsilateral IR muscle [8]. In thyroid-associated ophthalmopathy, which is a disease characterized by IR contracture, corrective effects in vertical deviation are larger compared to those of 
other patients with strabismus [16].Late overcorrection after IRR occurs at a high rate in thyroid-associated ophthalmopathy (50\%) [13], although this phenomenon sometimes also occurs in other vertical strabismus [19]. Maruo et al. reported that the rate in IRR for SOP was approximately 10\% [20]. Furthermore, the vertical deviation in the primary position was more than that in the downward and upward gaze positions in the present study, which may imply that contracture of the contralateral IR and ipsilateral superior rectus muscle induced the infraduction and supraduction limits, respectively. Hence, we considered that the cause of the high corrective effect in the present study was more likely to be late overcorrection with higher frequency than late undercorrection because the inverted Brown pattern that causes late undercorrection, in which vertical deviations in the downward and contralateral gaze positions are greater than those in the primary position, was not frequently encountered in the present study [21, 22].

The correlation analysis revealed that the corrective effect of IRR was negatively correlated with the preoperative difference in vertical deviation between the contralateral and primary gaze positions, although this relationship was no longer significant after excluding an outlier. This difference represents clinical IO-OA; thus, higher IO-OA produced a smaller corrective effect. Kishimoto et al. reported that the surgical effect of IOR was similar between patients with paretic and non-paretic eye fixation [23]. Therefore, for IOR, it is unnecessary to consider eye fixation. Although the reason for this negative correlation is unclear, IOR may be more appropriate in cases with high IO-OA, even for patients with paretic eye fixation.

Motor success was achieved in $70 \%$ and $55 \%$ in the primary and downward gaze positions, respectively, and reoperation was necessary in $15 \%$ of patients in the present study. In four patients with $1^{\circ}$ greater preoperative vertical deviation in the downward gaze position than in the primary gaze position, the motor success rate in the primary position was $100 \%$ and reoperation rate was $0 \%$. A previous study reported that the reoperation rate of IRR was higher than that of IOR (16\% vs. $7.6 \%)$ [15]. We believe that the corrective effect of IRR is not constant, as shown in Figure 2, which differs from the preoperative surgical table. We found that the corrective effect of IRR was larger in cases with less preoperative vertical deviation in the downward gaze position than in the primary gaze position. These results suggest that IRR could be performed in cases with $1^{\circ}$ more preoperative vertical deviation in the downward gaze position than in the primary gaze position by conventional $3(\Delta / \mathrm{mm})$ based on the targeted vertical deviation. However, in the other cases, it would be better to calculate the distance of surgery according to the difference of vertical deviation between the downward and primary gaze positions or select other procedures. 
This study had several limitations. First, due to its retrospective nature, this study was not carried out in accordance with a predetermined protocol; thus, there may have been a bias in choosing the surgical procedures. Future prospective studies, in which the distance of recession is determined before surgery using a regression line calculated from the results of our present study, could provide further evidence to support our findings. Second, we did not utilize MRI for evaluating atrophy of the superior oblique (SO) muscle or contracture of the IR muscle, which are both factors that could have influenced our results. For example, previous studies have reported atrophy or hypoplasia of the SO muscle belly [24-30] and contracture of the IR muscle [8] in patients with acquired SOP and those with congenital SOP. A subsequent study that investigates the correlation between the abnormal MRI features within the SO and IR muscles with the corrective effect of IRR may provide a more accurate prediction algorithm for vertical deviation before performing surgery. Third, we could not obtain patient data, including measurements vertical deviation, in the early postoperative phase. Because most of our patients were introduced from distant hospitals throughout Japan, they often returned to the original hospitals for follow-up in the early postoperative phase.

In conclusion, the important predictive factors that were associated with the corrective effect of IRR on vertical deviation were the preoperative difference in vertical deviation between the primary and downward gaze positions and the presence of preoperative stereopsis. Therefore, our findings suggest that these factors should be assessed prior to performing IRR for congenital SOP, particularly with paretic eye fixation.

\section{Funding:}

The Japanese Ministry of Education, Science, Sports, Culture and Technology provided financial support in the form of a grant (No. 26861451). The sponsor had no role in the design or conduct of this research.

\section{Conflict of interest:}

All authors certify that they have no affiliations with or involvement in any organization or entity with any financial interest (such as honoraria; educational grants; participation in speakers' bureaus; membership, employment, consultancies, stock ownership, or other equity interest; and expert testimony or patent-licensing arrangements), or non-financial interest (such as personal or professional relationships, affiliations, knowledge or beliefs) in the subject matter or materials discussed in this manuscript. 


\section{Ethical approval:}

All procedures performed in studies involving human participants were in accordance with the ethical standards of the institutional and/or national research committee and with the 1964 Helsinki declaration and its later amendments or comparable ethical standards. For this type of study formal consent is not required. 


\section{REFERENCES}

1. Knapp P (1971) First Annual Richard G. Scobee Memorial Lecture. Diagnosis and surgical treatment of hypertropia. Am Orthopt J 21: 29-37

2. Knapp P, Moore S (1976) Diagnosis and surgical options in superior oblique surgery. Int Ophthalmol Clin 16: 137-149

3. von Noorden GK, Murray E, Wong SY (1986) Superior oblique paralysis. A review of 270 cases. Arch Ophthalmol 104: 1771-1776

4. Helveston EM, Mora JS, Lipsky SN, Plager DA, Ellis FD, Sprunger DT, Sondhi N (1996) Surgical treatment of superior oblique palsy. Trans Am Ophthalmol Soc 94: 315-334

5. Mahmoud TA, Flanders M (2009) Treatment of Knapp Class V superior oblique palsy with contralateral inferior rectus muscle recession. Can J Ophthalmol 44: 320-322

6. Kushner BJ (2010) Vertical rectus surgery for knapp class II superior oblique muscle paresis. Arch Ophthalmol 128: 585-588

7. Miyata M, Shira Y, Kono R, Hamasaki I, Hasebe S, Ohtsuki H (2013) Effects of vertical muscle surgery on differences in the orientation of Listing's plane in patients with superior oblique palsy. Graefes Arch Clin Exp Ophthalmol 251: 2437-2443

8. Jiang L, Demer JL (2008) Magnetic Resonance Imaging of the Functional Anatomy of the Inferior Rectus Muscle in Superior Oblique Muscle Palsy. Ophthalmology 115: 2079-2086

9. Dickey CF, Scott WE, Cline RA (1988) Oblique muscle palsies fixating with the paretic eye. Surv Ophthalmol 33: 97-107

10. Mahmoud TA, Flanders M (2009) Treatment of Knapp Class V superior oblique palsy with contralateral inferior rectus muscle recession. Can J Ophthalmol 44: 320-322

11. Kushner BJ (1989) Errors in the three-step test in the diagnosis of vertical strabismus. Ophthalmology 96: 127-132

12. Helveston EM, Ellis FD (1983) Superior oblique tuck for superior oblique palsy. Aust J Ophthalmol 11: 215-220

13. Sprunger DT, Helveston EM (1993) Progressive overcorrection after inferior rectus recession. J Pediatr Ophthalmol Strabismus 30: 145-148

14. Ohtsuki H, Hasebe S, Kono R, Shiraga F (1999) Prognostic factors for successful surgical outcome with preoperative prism adaptation test in patients with superior oblique palsy. Acta Ophthalmol Scand 77: 536-540

15. Wang Q, Flanders M (2016) Surgical Management of Unilateral Superior Oblique Palsy: Thirty Years of Experience. Am Orthopt J 66: 79-86 
16. Peragallo JH, Velez FG, Demer JL, Pineles SL (2013) Postoperative drift in patients with thyroid ophthalmopathy undergoing unilateral inferior rectus muscle recession. Strabismus 21: 23-28

17. Simonsz HJ, Crone RA, van der Meer J, Merckel-Timmer CF, van Mourik-Noordenbos AM (1985) Bielschowsky head-tilt test-I. Ocular counterrolling and Bielschowsky head-tilt test in 23 cases of superior oblique palsy. Vision Res 25: 1977-1982

18. Schild AM, Fricke J, Neugebauer A (2013) Inferior rectus muscle recession as a treatment for vertical diplopia following cataract extraction. Graefes Arch Clin Exp Ophthalmol 251: 189-194

19. Wright KW (1996) Late Overcorrection after Inferior Rectus Recession. Ophthalmology 103: 1503-1507

20. Maruo T, Iwashige H, Kubota N, Sakaue T, Ishida T, Honda M, Hayashi T, Usui C (1996) Long-term results of surgery for superior oblique palsy. Jpn J Ophthalmol 40: 235-238

21. Awadein A, Pesheva M, Guyton DL (2006) "Inverted Brown pattern": a tight inferior oblique muscle masquerading as a superior oblique muscle underaction--clinical characteristics and surgical management. J AAPOS 10: 565-572

22. Marsh JD, Yilmaz PT, Guyton DL (2016) Deficiency of depression in adduction: clinical characteristics and surgical management of the "inverted Brown pattern". J AAPOS 20: 7-11

23. Kishimoto F, Hasebe S, Ohtsuki H (2011) Effects of inferior oblique muscle-weakening surgery on the Bielschowsky head-tilt phenomenon in patients with superior oblique palsy habitually fixating with the paretic eye. Jpn J Ophthalmol 55: 525-533

24. Horton JC, Tsai RK, Truwit CL, Hoyt WF (1990) Magnetic resonance imaging of superior oblique muscle atrophy in acquired trochlear nerve palsy. Am J Ophthalmol 110: 315-316

25. Demer JL, Miller JM (1995) Magnetic resonance imaging of the functional anatomy of the superior oblique muscle. Invest Ophthalmol Vis Sci 36: 906-913

26. Ozkan SB, Aribal ME, Sener EC, Sanac AS, Gurcan F (1997) Magnetic resonance imaging in evaluation of congenital and acquired superior oblique palsy. J Pediatr Ophthalmol Strabismus 34: 29-34

27. Sato M, Yagasaki T, Kora T, Awaya S (1998) Comparison of muscle volume between congenital and acquired superior oblique palsies by magnetic resonance imaging. Jpn J Ophthalmol 42: 466-470 
28. Kono R, Demer JL (2003) Magnetic resonance imaging of the functional anatomy of the inferior oblique muscle in superior oblique palsy.

Ophthalmology 110: 1219-1229

29. Demer JL, Poukens V, Ying H, Shan X, Tian J, Zee DS (2010) Effects of intracranial trochlear neurectomy on the structure of the primate superior oblique muscle. Invest Ophthalmol Vis Sci 51: 3485-3493

30. Shin SY, Demer JL (2015) Superior Oblique Extraocular Muscle Shape in Superior Oblique Palsy. Am J Ophthalmol 159: 1169-1179 


\section{FIGURE LEGENDS}

FIGURE 1. Box plot of corrective effect of inferior rectus recession on vertical deviation

The median corrective effect of inferior rectus recession on vertical deviation was $2.1 \% \mathrm{~mm}$. The interquartile range (IQR) was $1.1-3.4 \% \mathrm{~mm}$. The bars represent the range from the lower quartile $-1.5 \times$ IQR to the upper quartile $+1.5 \times$ IQR. An outlier was detected: one patient with a corrective effect of $7.3^{\circ} / \mathrm{mm}$.

FIGURE 2. Correlation between the corrective effect of inferior rectus recession on vertical deviation and the difference in preoperative vertical deviation in the primary and downward gaze positions

The difference in preoperative vertical deviation in the primary and downward gaze positions was associated with the corrective effect of inferior rectus recession on vertical deviation $(\% / \mathrm{mm})$ (the latter minus the former; $P=0.004, r=-0.61)$. The broken line represents the regression line $(y=-0.193 x+2.06)$. 
FIGURE 1

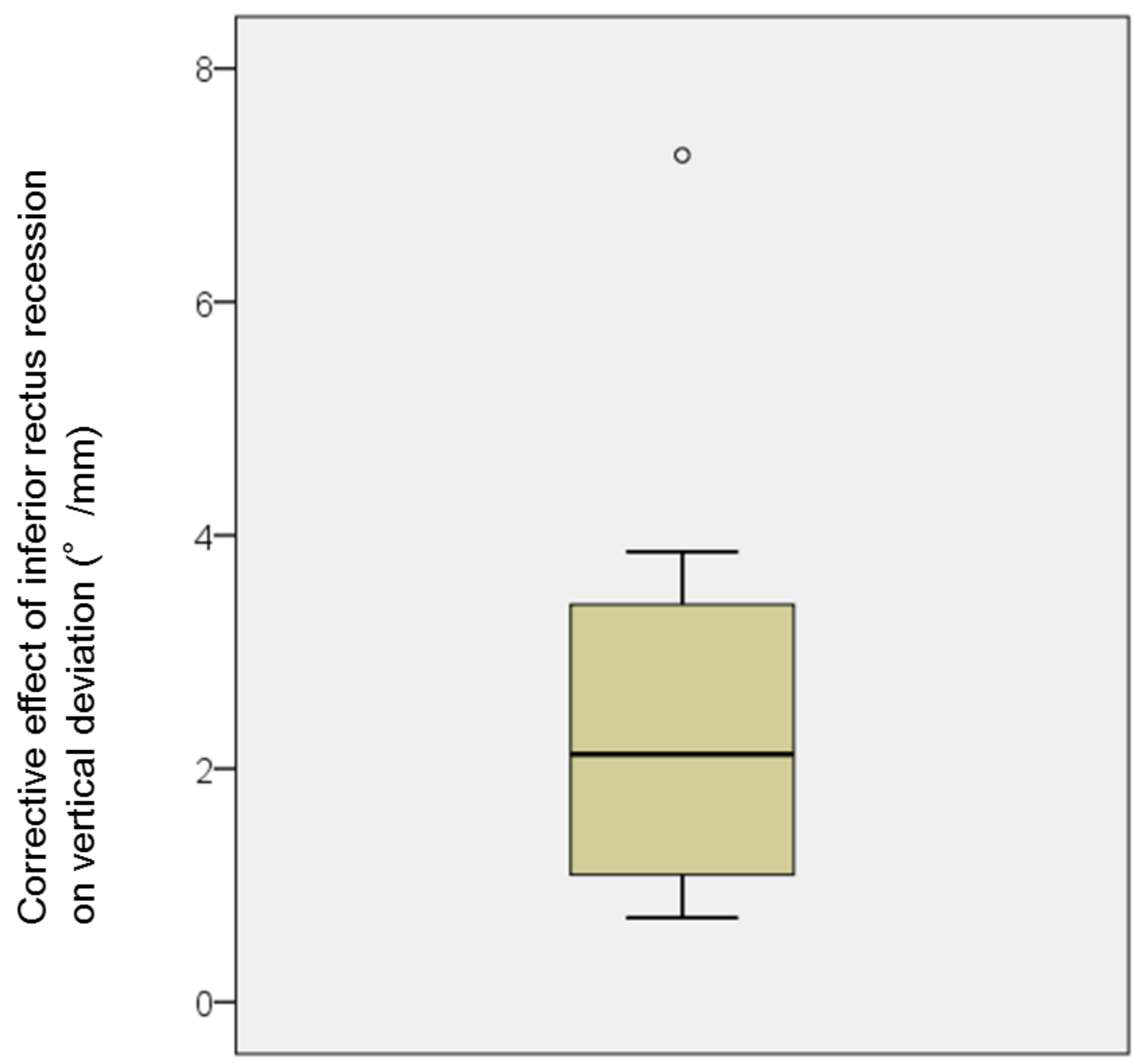




\section{FIGURE 2}

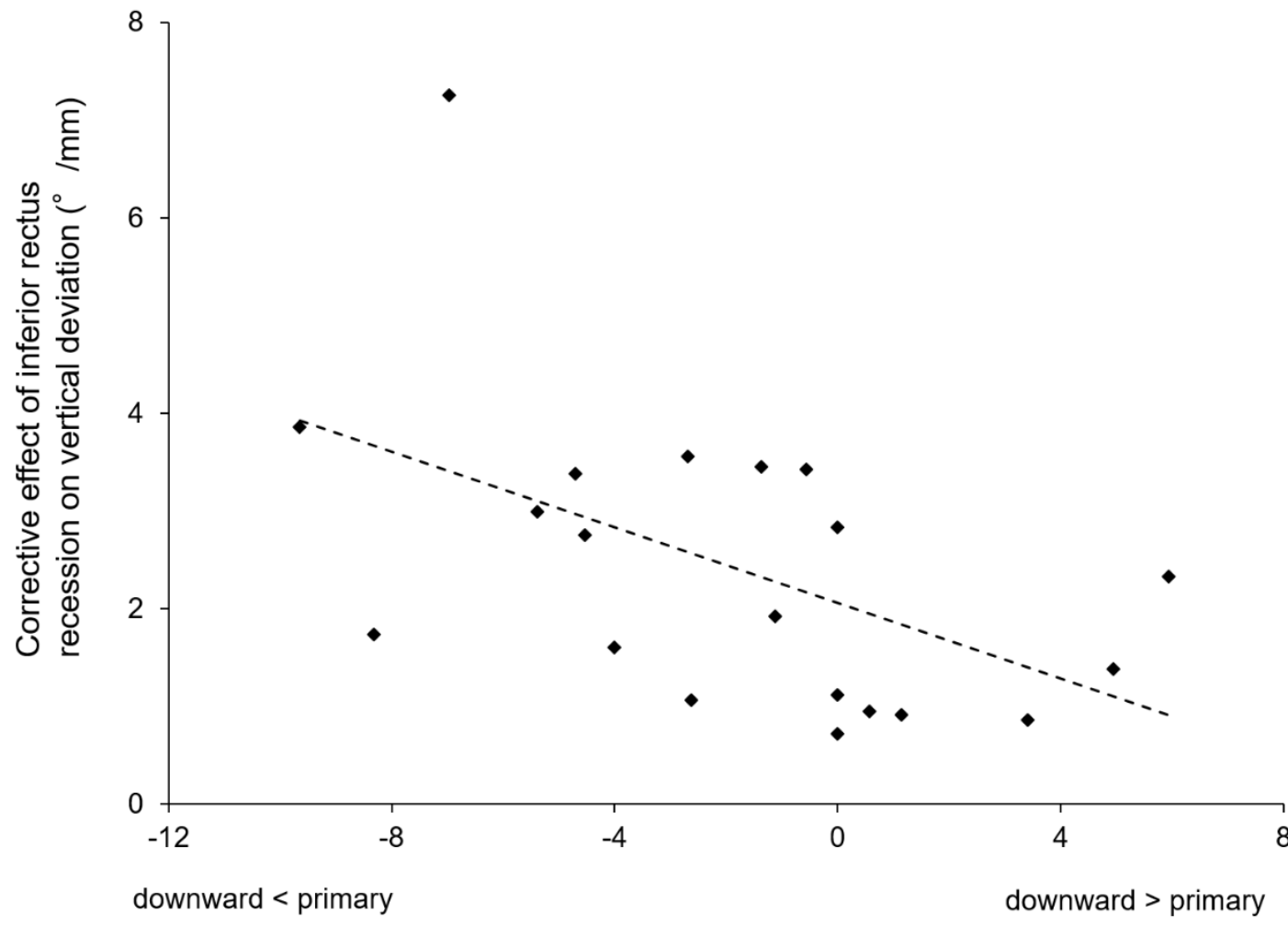

Subtraction of preoperative vertical deviation in the downward gaze position from that in the primary gaze positions $\left({ }^{\circ}\right.$ ) 
Table 1. Characteristics of the Study Population

\begin{tabular}{|c|c|c|}
\hline \multicolumn{2}{|l|}{$\mathrm{n}$, patients } & 20 \\
\hline \multicolumn{2}{|c|}{ Female sex, patients (rate) } & $10(50 \%)$ \\
\hline \multicolumn{2}{|l|}{ Age, years (range) } & $58.8 \pm 18.7$ (6 to 79$)$ \\
\hline \multicolumn{2}{|c|}{ Paretic eye fixation, patients (rate) } & $17(85 \%)$ \\
\hline \multicolumn{2}{|l|}{ LogMAR VA } & $-0.039 \pm 0.17$ \\
\hline \multicolumn{2}{|c|}{ Presence of preoperative stereopsis, patients (rate) } & $7(35 \%)$ \\
\hline \multicolumn{2}{|c|}{ Distance of recession, $\mathrm{mm}$} & $3.7 \pm 1.0$ \\
\hline \multicolumn{2}{|c|}{ Duration between surgery and measurement (days) } & $355 \pm 88$ \\
\hline \multirow{7}{*}{$\begin{array}{l}\text { Preoperative } \\
\text { vertical deviation } \\
\text { at } 5 \mathrm{~m},^{\circ}\end{array}$} & primary gaze position & $10.9 \pm 5.4$ \\
\hline & upward gaze position & $8.0 \pm 5.8$ \\
\hline & downward gaze position & $9.1 \pm 6.0$ \\
\hline & contralateral gaze position & $10.3 \pm 5.6$ \\
\hline & ipsilateral gaze position & $9.0 \pm 5.7$ \\
\hline & head tilted to the contralateral side & $7.5 \pm 4.8$ \\
\hline & head tilted to the ipsilateral side & $12.0 \pm 5.0$ \\
\hline \multicolumn{3}{|c|}{$\begin{array}{l}\text { Presence of preoperative stereopsis indicates that patients have stereoacuity } \leq 1980 \\
\text { sec of arc, as measured with the TNO stereo test. } \\
\text { logMAR VA = logarithm of the minimal angle of resolution }\end{array}$} \\
\hline
\end{tabular}


Table 2. Correlations Between the Corrective Effect of Inferior Rectus Recession on Vertical Deviation and Preoperative Study Parameters

\begin{tabular}{|c|c|c|c|c|c|}
\hline & \multicolumn{2}{|c|}{$\begin{array}{c}\text { All analysis } \\
(\mathrm{n}=20)\end{array}$} & \multicolumn{2}{|c|}{$\begin{array}{l}\text { Analysis except } \\
\text { an outlier }(n=19)\end{array}$} \\
\hline & & $P$ & r & $P$ & r \\
\hline \multicolumn{2}{|l|}{ Age (years) } & 0.07 & - & 0.10 & - \\
\hline \multicolumn{2}{|l|}{ Sex } & 0.83 & - & 0.88 & - \\
\hline \multicolumn{2}{|l|}{ LogMAR VA } & 0.22 & - & 0.39 & - \\
\hline \multicolumn{2}{|c|}{ Preoperative stereopsis (0: present, 1 : absent) } & $0.02^{*}$ & -0.51 & $0.049 *$ & -0.46 \\
\hline \multicolumn{2}{|c|}{ Distance of recession (mm) } & 0.86 & - & 0.98 & - \\
\hline \multicolumn{2}{|c|}{ Duration between surgery and measurement (days) } & 0.06 & - & 0.17 & - \\
\hline \multirow{7}{*}{$\begin{array}{l}\text { Preoperative } \\
\text { vertical deviation } \\
\text { at } 5 \mathrm{~m},{ }^{\circ}\end{array}$} & primary gaze position & 0.33 & - & 0.40 & - \\
\hline & upward gaze position & 0.30 & - & 0.46 & - \\
\hline & downward gaze position & 0.38 & - & 0.51 & - \\
\hline & contralateral gaze position & 0.65 & - & 0.64 & -- \\
\hline & ipsilateral gaze position & 0.61 & - & 0.78 & - \\
\hline & head tilted to the contralateral side & 0.78 & - & 0.79 & - \\
\hline & head tilted to the ipsilateral side & 0.82 & - & 0.94 & - \\
\hline \multirow{4}{*}{$\begin{array}{l}\text { Difference in } \\
\text { vertical deviation } \\
\text { to the primary } \\
\text { gaze position }\left(^{\circ}\right)\end{array}$} & upward gaze position & 0.99 & - & 0.54 & - \\
\hline & downward gaze position & $0.004^{*}$ & -0.61 & $0.01^{*}$ & -0.56 \\
\hline & contralateral gaze position & $0.03^{*}$ & -0.48 & 0.07 & - \\
\hline & ipsilateral gaze position & 0.52 & - & 0.74 & - \\
\hline \multicolumn{2}{|c|}{$\begin{array}{l}\text { Difference in vertical deviation during head-tilting } \\
\text { between the contralateral and ipsilateral sides }\left(^{\circ}\right)\end{array}$} & 0.80 & - & 0.99 & - \\
\hline \multicolumn{6}{|c|}{$\begin{array}{l}\text { Presence of preoperative stereopsis indicates that patients have stereoacuity } \leq 1980 \text { sec of arc, as } \\
\text { measured with the TNO stereo test. } \\
\text { logMAR VA = logarithm of minimal angle of resolution visual acuity; } \\
{ }^{*} \text { statistically significant }(P<0.05)\end{array}$} \\
\hline
\end{tabular}

